\title{
Sistemas de acceso a la información de prensa digital: tipología y evolución
}

\author{
Javier Guallar * \\ Ernest Abadal ** \\ Lluís Codina ***
}

Artículo recibido:

7 de noviembre de 2012.

Artículo aceptado:

12 de abril de 2013.

\section{Resumen}

El objetivo de este trabajo es clasificar y analizar los principales productos y servicios de información que facilitan el acceso a la prensa digital y a sus contenidos y mostrar su evolución en los últimos cinco años. El criterio fundamental para establecer la tipología de productos ha sido la función principal que realizan para el usuario, lo que nos ha llevado a establecer cuatro grandes ámbitos: acceso a cabeceras de prensa, búsqueda de información, seguimiento de información y agregación de contenidos. De cada una de estas clases, se describen y analizan distintas subtipologías: directorios, hemerotecas históricas, hemerotecas digitales

* Universidad de Barcelona; Universidad Ramon Llull, España. jguallar@gmail.com

** Universidad de Barcelona, España. abadal@ub.edu

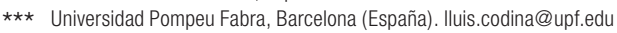


de medios, bases de datos de prensa, seguimiento de prensa, seguimiento en línea, seguimiento en redes sociales, agregadores manuales, agregadores automatizados y agregadores sociales. Se muestran para cada tipología algunos ejemplos significativos de las áreas lingüísticas inglesa y española.

Palabras clave: Prensa digital; Diarios digitales; Fuentes de información; Clasificaciones; Directorios; Hemerotecas; Bases de datos de prensa; Seguimiento; Agregadores.

\section{Abstract}

Digitized print media information access systems: typology and development

Javier Guallar, Ernest Abadal and Lluís Codina

The goal of this article is to classify and analyze the main products and services that provide access to online print media contents. The main factor determining the product type resides in a definition of the product's primary user function. Under this lens, four primary access function classes were established: 1) access to newspapers; 2) information search; 3) information clippings, and 4) content aggregation. For each of these classes, several sub-types are described and analyzed, including directories, newspaper archives, historical press archives, press data bases, press clippings, online clipping, social network clipping, manual and automatic aggregators, and social aggregators. The article also provides several illustrative Spanish- and English-language examples.

Keywords: Online press; Online newspapers; Information sources; Classifications; Directories, Press archives; Press databases; Clipping; Aggregators.

\section{INTRODUCCIÓN} incremento de la cantidad de información de prensa accesible en la red es constante y parece no tener fin. Nunca como ahora había sido posible poder consultar tanta información de actualidad, en todos los idiomas y, 
además y muy especialmente, de todas las épocas. Por otro lado, los perfiles de usuario interesados por estos contenidos son muy diversos: periodistas de investigación e historiadores que necesitan información de hemeroteca, cargos públicos y privados que siguen diariamente las noticias de su organización y de la competencia, gran público que está suscrito a sus temáticas de interés, etcétera.

Esta abundancia de contenidos y la diversidad de demandas, no obstante, generan algunas dificultades para la localización de informaciones concretas y precisas. ¿Dónde se pueden encontrar los periódicos o noticias sobre temas concretos que pueden interesar a un usuario específico en un momento determinado?

Además, en el nuevo ecosistema informativo digital de la Web, conviven junto a los medios de comunicación tradicionales en Internet y los diarios nativos digitales otros productos con contenidos periodísticos o informativos, como agregadores, bases de datos, productos de seguimiento o los denominados "medios sociales" (social media).

Deuze ha utilizado hace tiempo la expresión online journalisms (en plural) para referirse a este hecho. Ya no existe un único periodismo digital, sino varios periodismos, y establece una tipología de cuatro tipos de sitios web con contenidos periodísticos: 1) sitios web de noticias tradicionales; 2) directorios y agregadores de noticias; 3 ) sitios sobre los medios y de comentario y 4) sitios de discusión (Deuze, 2003: 10).

El trabajo de Deuze es un buen ejemplo de la preocupación de los estudiosos del ámbito por intentar aclarar, identificar y categorizar adecuadamente este panorama, de creciente complejidad. Así por ejemplo, en España se han publicado algunas interesantes recopilaciones y clasificaciones de recursos y fuentes de información sobre medios y periodismo digital. Es el caso de los estudios de autores como: Fuentes, Jiménez y González (2001), Rubio Lacoba (2002), Giménez Toledo (2002) y Giménez Toledo y López Martín (2003), Pareja Pérez (2003 y 2006), Arquero y García Ochoa (2005), Guallar y Abadal (2009b) y Abadal y Guallar (2010, cap. 2), Sánchez Vigil, Marcos Recio y Olivera Zaldua (2011) o Ivars-Nicolás (2012).

También cabe citar los estudios sobre grupos de productos concretos, como Jiménez (2003) y Díaz Noci (2004) sobre el sector del clipping, Renedo (2006) sobre los llamados “buscadores de noticias”, González (2009) sobre las bases de datos de prensa o Jiménez López (2002), Guallar y Abadal (2009a, 2010) y Guallar, Abadal y Codina (2012) sobre las hemerotecas de la prensa digital.

El estudio de las fuentes de información sobre prensa, por tanto, es un ámbito con notables variaciones, tanto en lo que se refiere a la tipología como a los productos concretos. Es por ello que entendemos que está plenamente justificado presentar nuevas revisiones y actualizaciones. 
El objetivo de este trabajo es presentar una categorización y un análisis de las principales fuentes de información que facilitan el acceso y la consulta a los diarios digitales y a sus contenidos, así como mostrar su evolución en los últimos cinco años.

En lo que se refiere a la categorización, se ha partido de dos trabajos anteriores (Guallar y Abadal, 2009b; Abadal y Guallar, 2010) que establecían una clasificación de las fuentes de información de prensa basada en la función que realizan (acceso a cabeceras, búsqueda, seguimiento y agregación de informaciones). En el presente texto se actualiza la tipología de productos que facilitan el acceso a contenidos de prensa, incluyendo nuevas categorías (como los sistemas de seguimiento en redes sociales y una subtipología en los agregadores automatizados) y revisando la orientación del resto.

De cada una de las categorías se incluyen algunos ejemplos de las áreas lingüísticas inglesa y española. Se trata de modelos que consideramos representativos y que tienen por función hacer más claras y explícitas las categorías. En ningún caso se persigue la exhaustividad.

El segundo objetivo del texto es mostrar la evolución de estos productos y servicios en los últimos cinco años. Es decir, se trata de valorar cuál es la importancia relativa de cada uno de ellos en el contexto de fuentes de información de prensa. ¿Cuáles están aún en crecimiento, cuáles han conseguido la consolidación y cuáles están empezando a caer en declive o en desuso?

La metodología ha consistido, en primer lugar, en una amplia revisión bibliográfica de las publicaciones sobre prensa digital y, especialmente, de fuentes de información sobre prensa. Por otro lado, se ha llevado a cabo un minucioso análisis de contenido sobre los ejemplos seleccionados de las fuentes de información sobre prensa para conocer sus funciones, estructura y características. De cada tipología se han establecido un grupo de indicadores que han servido para diferenciar con mayor claridad los perfiles de cada una de las categorías. Estos indicadores son: la cobertura de medios (uno o diversos), el alcance temporal, el costo (gratuito o de pago), los formatos de presentación del documento (HTML, texto, PDF) y la situación respecto de los derechos de explotación.

\section{TiPOLOGÍA DE FUENTES DE INFORMACIÓN}

Nuestra propuesta utiliza como criterio discriminador la función realizada por cada una de las fuentes de información, lo que nos lleva a establecer cuatro grandes áreas: 
- Acceso a cabeceras de prensa: servicios que facilitan el acceso a un determinado periódico o medio de comunicación. Son los directorios especializados en prensa.

- Búsqueda de información: sistemas que facilitan la consulta de información periodística de actualidad o retrospectiva. Son las hemerotecas históricas, las hemerotecas digitales de los medios y las bases de datos de prensa.

- Seguimiento de información: servicios que entregan periódicamente a sus usuarios recopilaciones personalizadas de información de actualidad. Son los sistemas de seguimiento de prensa tradicionales y los servicios de seguimiento en línea. A estos sistemas se les puede añadir un tercero de aparición reciente: el seguimiento en redes sociales.

- Agregación de contenidos: sistemas que recopilan información de actualidad publicada por múltiples medios. Se distingue entre agregadores manuales, automatizados y sociales.

Para cada categoría se ofrece una explicación de la función que ejercen, se presentan sus características principales, se muestran algunos ejemplos representativos de las dos áreas lingüísticas analizadas: inglesa y española, y se analiza su evolución.

\section{Acceso a medios (directorios)}

El principal objetivo de este tipo de recursos es facilitar el acceso a un determinado periódico o medio de comunicación. Se trata de recursos que nos conducen hasta la puerta de los medios y que, salvo excepciones, no permiten consultar directamente el contenido (las noticias).

Son los directorios de medios de comunicación un tipo de publicación con larga trayectoria en el entorno impreso y que sigue manteniendo vigencia en Internet, aun cuando en este caso su uso sea casi siempre como complemento a la consulta en buscadores.

El sistema de organización más utilizado en los directorios es la agrupación de títulos de prensa por países y por tipo de medio de comunicación (prensa, radio y televisión). Los datos que normalmente se ofrecen de cada medio no son muy extensos; en general, título y URL, aunque a veces se incluyen también otros (país, idioma, etc.). Mostramos dos ejemplos representativos:

- Kidon Media Link

http://www.kidon.com/media-link/index.shtml

Directorio de medios de comunicación muy completo y práctico. Uno 
de los de mayor calidad. Se puede acceder a los medios por continentes, países, lenguas y por tipo de medio (agencias, periódicos, revistas, radio o televisión). Ofrece acceso a unas 20000 fuentes de prensa de todo el mundo, por ejemplo, a más de 9000 de Estados Unidos, 601 de Reino Unido, 355 de Argentina, 317 de España o 254 de México.

- Kiosko http://kiosko.net/

Directorio español muy visual de prensa española nacional, regional y local, junto a una selección de prensa internacional en aumento. En total, algo menos de 1000 diarios. Además de dar acceso a los sitios web de los diarios, muestra una imagen de las portadas de la edición impresa de esos periódicos. Su principal atractivo es la visualización rápida de las portadas de los medios de un país, de un continente o de un ámbito temático (prensa de información general, económica o deportiva).

En lo que se refiere a su evolución, se trata de un tipo de fuente de información que no ha sufrido cambios en los últimos años y, al parecer, no tiene mucho más recorrido. No han aparecido nuevos productos y su interés actual es más bien reducido o escaso, en consonancia con la información que apor$\tan$.

\section{Búsqueda de información}

Las fuentes especializadas en la búsqueda mediante recuperación de información permiten localizar una noticia concreta aparecida en uno o en diversos medios, ya sea del día o retrospectiva. Se puede distinguir entre las hemerotecas históricas, con fondos antiguos digitalizados de varias publicaciones; las hemerotecas digitales de un medio, que permiten la consulta a los fondos impresos y digitales de un diario; y las bases de datos de prensa, con noticias de múltiples medios.

\section{Hemerotecas históricas}

Son servicios en los que se ha llevado a cabo una digitalización de las páginas de diarios y revistas históricos, y se han puesto a disposición del público en Internet. Una buena parte de los impulsores de estas iniciativas son bibliotecas (nacionales, municipales, etc.) que cuentan con el respaldo económico de las organizaciones que las sustentan, ya sean ministerios, ayuntamientos $u$ otras administraciones. Los formatos más habituales de presentación de los documentos son dos: la imagen gráfica de las páginas escaneadas (por ejemplo, 
en JPG) y el formato PDF, en ocasiones con opción de búsqueda de palabras dentro de los documentos.

Son fuentes muy útiles en investigaciones y búsquedas de informaciones de contenido histórico. La cobertura geográfica puede ir desde la ciudad a la provincia, la comunidad autónoma, el país e incluso más allá, como sucede en la Biblioteca Cervantes, en varios países de América Latina. Se da el caso de diversos títulos que pueden formar parte de más de una hemeroteca histórica.

- Chronicling America

http://www.loc.gov/chroniclingamerica/

Estados Unidos es el país que cuenta con mayor número de proyectos de hemerotecas históricas en marcha. Entre los diversos existentes, uno de los más amplios y activos es el National Digital Newspaper Program gestionado en los EUA por el National Endowment for Humanities y que cuenta con la asistencia de la Library of Congress (http://www.neh.gov/grants/guidelines/ndnp.html). La mayoría de las digitalizaciones realizadas proceden de microfilm. El periodo incluido va de 1836 a 1922. Chronicling America es el portal que permite consultar todos los periódicos del programa de forma conjunta.

- Biblioteca Virtual de Prensa Histórica

http://prensahistorica.mcu.es/prensahistorica/es/consulta/busqueda.cmd Es la hemeroteca histórica más importante de ámbito español. Impulsada por el Ministerio de Cultura, ofrece el contenido de unos 2000 títulos de los siglos XVIII-XX. Según los datos de su sitio web, en marzo de 2010 contenía cinco millones de páginas digitalizadas. El formato de visualización era en principio JPG, ampliado posteriormente a PDF, con opciones de búsqueda por palabras dentro de las páginas de los diarios.

- Hemeroteca Nacional Digital de México

http://www.hndm.unam.mx/

Se trata de una iniciativa de la UNAM que contiene más de nueve millones de páginas correspondientes a casi 1000 títulos de prensa editada en México y también en otros países. Una parte de los contenidos no se pueden consultar por Internet porque se trata de obra que aún no ha pasado al dominio público.

Los cambios producidos en los últimos años en este tipo de productos van en la dirección de ir completando los procesos de digitalización. Es predecible que a medio plazo se conseguirá disponer de la (casi) totalidad de la 
prensa histórica en formato digital, lo que constituirá un producto del máximo interés para historiadores e investigadores de prensa en general.

\section{Hemerotecas digitales de un medio}

La hemeroteca digital es uno de los servicios específicos del entorno digital que los diarios les ofrecen a sus usuarios y que los diferencian más claramente de las ediciones impresas. Es el servicio de los diarios en Internet que se identifica más claramente con la característica de la prensa digital denominada "memoria" o "documentación" (Palacios, 2009; Abadal y Guallar, 2010).

Resaltaremos aquí fundamentalmente su papel como fuente de información; es decir, desde la posición del usuario (especialmente el profesional) que necesita localizar una o varias informaciones publicadas por un medio de comunicación en concreto. Desde este punto de vista, son fuentes de información muy importantes, y en muchos casos, insustituibles. En este sentido, algunas cuestiones a tener en cuenta sobre estos servicios son su alcance temporal, el costo y las prestaciones de consulta.

Si desde los inicios de la prensa en Internet hasta el año 2002, aproximadamente, la mayoría de diarios ofrecían una hemeroteca en línea meramente "testimonial" de los últimos días o semanas, desde entonces la cantidad de fondos accesibles ha crecido de forma notable, y es claramente visible en los últimos años la tendencia por parte de las cabeceras de prensa más importantes del mundo a ofrecer la totalidad de su archivo en el sitio web del diario. Con todo, hay una gran diferencia en estos momentos entre los diarios que ofrecen grandes hemerotecas en línea, con la totalidad de sus fondos, y aquellos que siguen ofreciendo hemerotecas con muy pocos contenidos.

En cuanto al costo, la tendencia a ofrecer los contenidos de archivo gratuitos o de pago no está del todo clara, aunque la actual coyuntura de crisis haya agudizado la necesidad de cobro, y el equilibrio entre ambas opciones parezca decantarse cada vez más hacia los sistemas de pago, mediante diferentes fórmulas.

La consulta de las hemerotecas ha presentado tradicionalmente dos formas de acceso: la consulta de ejemplares anteriores íntegros por navegación cronológica (browsing) y el acceso mediante búsqueda (searching) por palabra clave utilizando un buscador. Ambos sistemas coexisten en estos momentos, si bien la preponderancia del acceso por buscador es cada vez más clara. La calidad de las prestaciones de consulta ha ido mejorando a lo largo de los últimos años, aunque en el caso de algunos diarios modestos o locales, los servicios de búsqueda pueden llegar a ser bastante deficientes. También se da la paradoja de que en ocasiones la tendencia de muchos sitios web hacia 
la simplificación de sus sistemas de búsqueda con el objeto de facilitar el proceso al usuario medio (por ejemplo, haciendo desaparecer las opciones de búsqueda avanzada o algunos operadores booleanos) haya supuesto, desde el punto de vista del uso profesional, un empobrecimiento y retroceso (Guallar, Abadal y Codina, 2012).

- The New York Times

http://www.nytimes.com/ref/membercenter/nytarchive.html

El diario norteamericano tiene un archivo en línea desde 1851. El servicio de archivo está dividido en dos bloques: 1851-1980 y 1981-actualidad, y cuenta con opciones de búsqueda avanzada y un completo sistema de ayuda. Es una de las hemerotecas de diarios de referencia a nivel internacional.

- El País

http://www.elpais.com/archivo/

- La Vanguardia

http://www.lavanguardia.com/hemeroteca/index.html

Las hemerotecas de los diarios españoles El País y La Vanguardia son dos ejemplos destacados del estado actual de estos servicios en la prensa digital, ambos de características y orientaciones bien diferenciadas. Las dos ofrecen la totalidad del fondo desde la fundación del diario: El País en 1976 y La Vanguardia en 1881. Combinan los contenidos gratuitos (en el primer caso el formato html, en el segundo, el contenido histórico menos los últimos días en formato pdf) con los contenidos de pago para suscriptores. Además, La Vanguardia destaca por la variedad de servicios y contenidos de la sección Hemeroteca, como efemérides, reportajes históricos, perfil en redes sociales y otros.

En esta tipología de productos, los grandes diarios disponen prácticamente ya de todos los fondos en Internet. En los últimos años la mayoría de grandes cabeceras ha completado la fase de digitalización de sus colecciones retrospectivas y las ofrecen a suscriptores y usuarios en general como complemento a la información diaria bajo algún esquema de comercialización. En cambio, buena parte de diarios regionales y locales tienen todavía pendiente esta tarea para los próximos años.

\section{Bases de datos de prensa}

Las bases de datos de prensa se caracterizan por realizar el análisis y la indización de las noticias aparecidas en diversos medios y por disponer de sistemas 
avanzados de consulta, que son superiores a los que ofrecen los propios medios (aunque las diferencias se hayan ido reduciendo con los años). Están dirigidas a un usuario profesional, no sólo por sus contenidos sino también y especialmente debido a su costo; además pueden ofrecer servicios de seguimiento de prensa. Adicionalmente, llevan a cabo sus servicios mediante acuerdos contractuales con los medios, con lo cual sus páginas de resultados proporcionan el acceso directo a los documentos y no a una lista de enlaces, como sucede con otras fuentes de información de prensa. En suma, se pueden considerar como los mejores sistemas de búsqueda existentes para localizar contenidos periodísticos.

Tienen una importante tradición e implantación en el ámbito anglosajón y han tenido una introducción más reciente en otras áreas. Las más importantes internacionalmente son Lexis Nexis y Factiva, diferenciadas claramente del resto; en español es relevante $M y$ News.

- Lexis Nexis

http://www.lexisnexis.com

Está considerada como la mayor base de datos comercial del mundo. Además de información de prensa, tiene información económica, fiscal y legislativa, con más de tres mil millones de documentos de unas 42000 fuentes de información. El apartado de noticias de actualidad incluye unas 9700 fuentes, de las cuales 2100 son diarios. Por ejemplo, en español el número de fuentes es superior a 400.

- Factiva

http://global.factiva.com

Creada en 1999 por la agencia de noticias Reuters, que anteriormente comercializaba Reuters Business Briefing, y la empresa Dow Jones. Además de información periodística, contiene información económica y financiera. Da acceso al contenido de unas 10000 fuentes, de las cuales unas 230 son en español.

- My News

www.mynews.es

My News es la base de datos con contenidos de prensa española más importante. Tiene también servicio de seguimiento de prensa. Creada en 1996 (desde 1998 en formato web), ofrece el texto completo y los PDF de 180 fuentes de prensa impresa y también de 450 fuentes exclusivamente digitales, con una cobertura temporal que puede llegar para algunos diarios hasta 1996. Los servicios de hemeroteca tienen las prestaciones documentales más altas entre los productos españoles, con tres opciones de búsqueda: simple, avanzada y profesional. Es 
probablemente la herramienta en documentación de prensa española más utilizada en el sector periodístico por profesionales de la información.

La base de datos de prensa es un producto dirigido a profesionales del sector de la comunicación (periodistas y documentalistas de empresas de medios), a gabinetes de prensa de empresas y a organismos de diversos ámbitos, incluyendo partidos políticos y administraciones públicas, por ejemplo. Aparece con pocos cambios en los últimos años, salvo mejoras continuadas en las interfaces de usuario, cada vez más amigables. Presenta pocas expectativas de variación a corto y medio plazo, representa una incógnita a largo plazo en un horizonte de plena digitalización de la noticia desde su misma creación y en el futuro ecosistema de la web móvil.

\section{Seguimiento}

Los productos de esta categoría tienen por objetivo fundamental la elaboración de recopilaciones temáticas de información de actualidad personalizadas para los usuarios, quienes las reciben a medida que se van produciendo. Se puede diferenciar entre servicios de seguimiento tradicionales de clipping y los más recientes servicios de seguimiento en línea. Por otra parte, la enorme popularización de los sitios web de redes sociales en Internet como $\mathrm{Fa}$ cebook y Twitter ha dado lugar en los últimos años a la generalización del uso de estos servicios también como sistemas de seguimiento de medios o de informaciones publicadas en Internet.

En general, suelen ser servicios más económicos que las bases de datos, si bien en el caso de los servicios de seguimiento tradicionales, éstos pueden tener casuísticas muy variadas. Los servicios de seguimiento tradicionales de clipping suelen tener en la actualidad acuerdos contractuales con los proveedores de la información, especialmente cuando se trata de contenidos de prensa impresa, aunque algunas empresas hayan tenido en el pasado enfrentamientos con los medios por su inexistencia. Por el contrario, los sistemas de seguimiento en línea (y de seguimiento en redes sociales) enlazan a las fuentes originales en Internet, por lo que no pueden garantizar que estos enlaces que aparecen en sus páginas de resultados se resuelvan con éxito. De hecho, pueden presentar una amplia casuística en forma de enlaces rotos, obsoletos, que exigen una suscripción, etc. No obstante, es evidente que cumplen una función y responden a un tipo de demanda concreta. 


\section{Seguimiento de prensa (clipping)}

Tradicionalmente, las empresas de clipping realizaban un seguimiento de las publicaciones impresas, seleccionaban informaciones a partir de las peticiones efectuadas por sus clientes y se las enviaban (Jiménez, 2003). Con la llegada de la información digital, el sector del clipping tradicional evolucionó y en la actualidad digitalizan las informaciones impresas o reciben directamente los documentos digitales de los medios mediante acuerdos con los mismos, a la vez que han añadido también en algunos casos seguimiento de medios en línea.

La relación entre medios de comunicación y servicios de clipping y resúmenes de prensa no ha estado exenta de enfrentamientos y tensiones a lo largo de los años. En estos momentos, la mayoría de empresas del sector llegan a acuerdos con los medios, a los que pagan un canon en función de las noticias que sirven a sus clientes.

El funcionamiento de estos servicios es simple. Un equipo de profesionales selecciona a diario las noticias para los clientes en función de las necesidades expresadas por éstos y les entregan los resultados mediante alertas enviadas por correo electrónico, habilitando espacios en sus webs para la consulta, o por el sistema tradicional de documentos impresos. En la mayoría de productos, los usuarios no pueden configurar personalmente sus perfiles temáticos o realizar búsquedas documentales retrospectivas (como permiten las bases de datos de prensa antes descritas), aunque los mejores ejemplos de esta categoría están ofreciendo servicios cada vez más variados.

- TNS

http://www.tnsglobal.com/

Taylor Nelson Sofres (TNS) es un grupo empresarial formado por la fusión de diversas compañías fundadas en los años sesenta del siglo pasado dedicadas a la investigación de mercados, como Taylor Nelson (Reino Unido), Intersearch (USA) o Sofres (Francia), que se ha fusionado a su vez con Research International (Reino Unido). Una de las muchas actividades que realiza TNS es el seguimiento de informaciones de medios de comunicación. En este campo lleva a cabo diferentes servicios personalizados, habitualmente en cuatro áreas (prensa, en línea, radio y televisión), con más de 10000 fuentes. Tiene delegaciones en muchos países.

- Acceso

http://www.acceso.com/

Acceso es la empresa más importante de clipping en español con sedes en España, Perú y Panamá. Se especializa en servicios de seguimiento 
de medios con cuatro áreas (prensa, Internet, radio y televisión) y en la elaboración de estudios de mercado y de informes hechos a medida para todo tipo de entidades. El desglose aproximado por tipos de medios es de unos 55000 medios en línea, 670 de prensa escrita, 48 de televisión y 25 de radio, y si se tienen en cuenta los medios sociales de los que hace seguimiento, se llega a unos dos millones de fuentes. La plataforma Acceso 360 le permite al usuario gestionar sus seguimientos y su comunicación y marketing o mercadeo.

Aunque el perfil de usuario no ha cambiado en los últimos años, se trata de un producto que ha ido incorporando mejoras tecnológicas de una forma continua, así como interfaces de usuario cada vez más amigables para facilitar la consulta y el acceso a los contenidos seleccionados, mientras que, en lo que se refiere a la cobertura ofrecida, está incorporando también diversas fuentes en línea (que son la especialidad del grupo siguiente).

\section{Seguimiento en línea}

Son servicios comerciales de seguimiento de fuentes en Internet, que surgen a comienzos del siglo XXI. Utilizan potentes sistemas de búsqueda que vuelcan y categorizan el contenido captado en sus bases de datos. El sistema permite acceder a múltiples fuentes de información con un ritmo de actualización constante.

No le muestran el texto completo de la noticia a sus clientes sino únicamente el enlace. Por ello, el handicap respecto a los servicios anteriores es que no permiten acceder directamente a contenidos de medios que sean de pago o tengan restringido su acceso mediante registro. Por el contrario, su principal ventaja es la enorme cantidad de medios digitales que pueden capturar.

- Moreover

http://www.moreover.com/

Fundado en 1998 y con oficinas en Gran Bretaña y Estados Unidos, es el referente y pionero de este tipo de productos a nivel internacional. Combina los servicios profesionales de pago dirigidos a empresas con los servicios gratuitos.

- iMente

www.imente.com

Producto español de seguimiento en línea creado en el año 2000, vacía una ingente cantidad de fuentes de Internet: en principio unas 40 000, que incluyen cada vez más blogs y medios sociales, llegando 
hasta un millón quinientos mil. Ofrece variados servicios personalizados de seguimiento, con consulta de resultados vía web o mediante alertas por correo electrónico, y un sistema de publicación de titulares en la Web. Otro producto de características muy similares en español es iConoce (www.iconoce.com).

El cambio más notable sufrido por los servicios de seguimiento en línea en los últimos años se ha producido en la cantidad de fuentes indexadas, que se han ido incrementando y diversificando cada vez más, hasta abarcar el conjunto de productos en línea que pueden ser de interés informativo para sus usuarios, por tanto, no ya solamente diarios digitales sino también blogs y medios sociales.

\section{Seguimiento en redes sociales}

Un nuevo sistema de seguimiento se está extendiendo con rapidez, de la mano de la generalización del uso de los sitios de redes sociales (SRS) más populares como Facebook (www.facebook.com) y Twitter (www.twitter.com), aparecidos ambos en 2006, y con una audiencia masiva desde finales de la primera década del siglo XXI. La posibilidad de seguir informaciones de medios de comunicación desde los perfiles personales en las redes sociales es ahora una actividad cada vez más extendida en Internet, heredera de las suscripciones RSS, que continúan existiendo.

En este caso no nos estamos refiriendo a productos a los que un usuario de Internet accede para obtener información de prensa, sino que son los propios perfiles de cada persona en las redes sociales los que le permiten hacer seguimientos de informaciones publicadas en Internet, las cuales son "compartidas" o "recomendadas" por otros perfiles a los que ese usuario sigue. Esos perfiles "recomendadores" pueden ser a su vez de otras personas o de entidades diversas, como los propios medios de comunicación, por ejemplo.

Así, un perfil en Twitter permite seguir (following) tanto a medios de comunicación en línea, que periódicamente publican mensajes cortos (tweets) con enlaces a sus noticias, como a otros usuarios de esa red social que pueden recomendar a sus contactos o seguidores aquellas informaciones que consideran de interés.

Además de los propios sitios de redes sociales, es notable la irrupción de numerosos servicios que permiten realizar el seguimiento o monitorización del contenido de estos SRS. Algunos de ellos son los productos de gestión de redes sociales, como Hootsuite (http://www.hootsuite.com) y Tweetdeck (http://www.tweetdeck.com/). Se trata de servicios especializados, entre 
otras opciones, en la gestión de diversos perfiles en redes sociales y en la monitorización de listas de usuarios y de búsquedas a partir de etiquetas o hashtags en Twitter. Otros productos a destacar son los especializados en el seguimiento de las informaciones de una determinada marca o perfil en los medios sociales, como es el caso de Social Mention (http://www.socialmention.com).

Se trata de una nueva categoría que ha irrumpido con enorme fuerza en el ámbito de las fuentes de información en los últimos cinco años y que no estaba considerada en los estudios anteriores. Representa el mayor factor de novedad en este ámbito y tiene un cierto potencial disruptivo, aunque aún resulta pronto para poder decidir sobre él.

\section{Agregación de información}

Denominamos aquí agregadores de noticias a aquellos productos que recopilan información del día publicada por múltiples medios y la agrupan en un sitio web. Se puede diferenciar entre agregadores manuales, automatizados y sociales. Son servicios gratuitos, que suelen ofrecer el titular y el enlace a noticias del día de diferentes medios; es decir, no suelen alojar el contenido íntegro de la noticia original (como los productos de búsqueda de información y los de seguimiento) sino solamente el enlace a la misma (como los servicios de seguimiento en línea).

Los agregadores manuales, de características generalmente modestas, habían existido desde los orígenes de Internet, pero la entrada de grandes empresas como Google y Yahoo en el sector mediante la creación de agregadores automatizados con contenidos de un gran número de fuentes ha originado polémicas y enfrentamientos con los medios, todavía no plenamente resueltos, que los acusan de hacer negocio a su costa. Finalmente, un nuevo tipo de agregadores surgidos en los últimos años son los que aquí denominamos agregadores sociales, que siguen las herramientas y la filosofía de la web 2.0.

\section{Agregación manual de noticias}

La presentación de contenidos de prensa seleccionados manualmente por un equipo de profesionales (usualmente periodistas y documentalistas) y sin la ayuda de sistemas automatizados ha existido desde los orígenes de la Web, pero la expansión de los sistemas basados en robots de búsqueda ha acabado marginando estos sistemas, dejando pocos ejemplos en la actualidad.

- Newser

www.newser.com 
Lanzado en Estados Unidos en 2007, es un excelente ejemplo de sitio web de agregación de noticias seleccionadas por un equipo de profesionales. Además de enlazar a las fuentes originales, el equipo de Newser redacta resúmenes de las noticias en pocos párrafos, algo que en la terminología actual de Internet se identifica también como news curation (curaduría de noticias o "curación" de noticias).

- Hago Clic http://www.hagoclic.com En español un buen ejemplo es Hago Clic, una web nacida en 2009 que pertenece al grupo editorial del diario La Vanguardia, cuyo contenido consiste en una selección de las noticias del día. Se presenta así: "El equipo editorial de HagoClic.com selecciona cada día los temas más destacados de la actualidad y escoge los enlaces más relevantes sobre los mismos".

La actividad de este tipo de agregadores se ha venido reduciendo progresivamente, y no parece que este producto vaya a ir a más en los próximos años, ni en cuanto a oferta de servicios ni tampoco en cuanto a demanda por parte de los usuarios. Se prevé un estancamiento a corto y medio plazo.

\section{Agregación automatizada de noticias}

A diferencia de los anteriores, que tienen una redacción que selecciona y presenta las noticias del día según criterios periodísticos, los sistemas automatizados se basan en medios exclusivamente tecnológicos, los denominados "buscadores de noticias", robots que rastrean Internet, extraen noticias de las webs de prensa y las clasifican y las presentan utilizando algoritmos informáticos, sin intervención humana.

- Google News

http://news.google.com/

http://news.google.es/

Google News, el buscador de noticias de Google aparecido en 2002, es el prototipo de agregador de noticias automatizado. Organiza y agrupa automáticamente noticias de unos 50000 medios en 30 lenguas, se publica en 72 ediciones y consigue mil millones de clics cada mes, una cuarta parte de los cuatro mil millones de clics que Google redirige mensualmente a los medios (Varela, 2012). En su versión en español rastrea unas 700 fuentes. La interfaz de Google News difiere de la del buscador generalista, ya que aquí no se trata de una caja 
de búsqueda sino de una web con aspecto de portal periodístico -de "diario de diarios"- donde, junto a las opciones de búsqueda, se muestran de manera agrupada los titulares de las noticias consideradas más relevantes con los enlaces a la página original en la que se ha publicado el contenido íntegro de la noticia. El sistema permite al usuario personalizar la página de inicio con sus secciones de interés, y dispone de otros servicios como la recepción de alertas por correo electrónico.

- Popurls

http://popurls.com

Creado en 2006, es un agregador que incluye de manera automatizada una selección de fuentes informativas de todo tipo y no sólo de prensa digital, por ejemplo, blogs, otros agregadores (Google News, Digg) o medios sociales (Flickr, Youtube).

- Abasto de noticias

http://www.abastodenoticias.com

Nacido -al igual que Google News- en 2002, este agregador es un ejemplo de alternativa modesta en español a los grandes sistemas internacionales, localizada en el ámbito informativo de un país. Cuenta con un sistema de categorización con 150 temáticas y ofrece servicio de titulares para webs.

- Flipboard

http://flipboard.com

Otro tipo de agregador automatizado diferente a los anteriores, y de aparición muy reciente, a partir de 2010-2011, es el nacido originalmente para dispositivos móviles como tabletas y smartphones. Coincide con los agregadores de la Web en la agrupación de noticias del día en un único lugar, pero se diferencia en que la agregación principal aunque no exclusiva es la fuente (el medio) y no la noticia (Guallar y Masip, 2012). Probablemente el ejemplo más destacado sea Flipboard, aparecido en 2010, y que se presenta como una "revista personalizada" que simula el hojeo de una revista de una manera excelente en la tableta.

Los agregadores automatizados representan otra de las grandes novedades en el ámbito de este estudio. Han experimentado un notable éxito en los últimos años y disponen de un enorme uso a partir de su incorporación en los equipos de la Web móvil, aunque por el momento la mayoría de estos servicios carece de la opción de búsqueda retrospectiva. Por este motivo, pese a su éxito parecen un producto de espectro amplio antes que un producto especializado. 


\section{Agregación social de noticias}

Los sistemas de promoción de noticias, conocidos también como agregadores sociales de noticias o sitios de filtrado social de noticias, son un tipo de productos de la denominada web social o web 2.0, que agrupan noticias en un sitio web.

Aquí son los usuarios quienes configuran los contenidos del sitio web. Éstos envían noticias u otras informaciones de su interés existentes en la Web enlazando la fuente original, y posteriormente son votadas o bien comentadas por otros usuarios, y pueden pasar a la página principal. Por tanto, son sistemas que permiten centralizar y valorar informaciones relevantes para una comunidad siguiendo los principios de colaboración e interacción característicos de la web social.

- Digg http://www.digg.com

El agregador social más veterano es Digg, aparecido en 2004, con noticias inicialmente especializadas en ciencia y tecnología, y posteriormente ampliadas a otras temáticas, como entretenimiento, negocios, deportes, etc. Fue el primer sitio en que los usuarios podían decidir las noticias de portada a partir de sus votos.

- Menéame http://meneame.net/

Menéame, nacido en 2005, goza de gran popularidad en la comunidad de usuarios de lengua española. De características similares a Digg, su diferencia principal es que los usuarios no registrados (por tanto, anónimos) también pueden votar las noticias, aunque no enviarlas. Menéame fue desarrollado en software libre y su creador liberó el código fuente, lo que ha facilitado su posterior clonación y adaptación a otros ámbitos.

Después de un relativo auge en la segunda mitad de la primera década del siglo XXI, la oferta de este tipo de productos se ha mantenido más o menos similar en los últimos años. No parece tampoco que vaya a incrementarse a corto o mediano plazo. Sin duda, el boom de los sitios de redes sociales y de otras fórmulas participativas en la Red ha puesto límites a lo que hubiera de relativa novedad en su planteamiento. 


\section{Conclusiones}

Vamos a referirnos a la categorización de fuentes de información, destacando cuáles son las principales diferencias entre los productos analizados y, en segundo lugar, vamos a referirnos a la evolución que han sufrido en los últimos cinco años.

La función de las fuentes de información de prensa digital (acceso a cabeceras, búsqueda, seguimiento y agregación de informaciones) es un criterio que consideramos especialmente útil para establecer una tipología de productos y clarificar un poco la amplia oferta de servicios existente en este sector.

Este criterio nos ha permitido establecer también una subtipología que ha sido descrita, analizada y ejemplificada en el apartado anterior. La Tabla 1 (página siguiente)intenta presentar una síntesis de las características diferenciales de cada una de las clases establecidas y así mostrar una visión de conjunto. Se tienen en cuenta la cobertura de medios incluidos (uno o varios), el alcance temporal (retrospectivo o de actualidad), el costo (gratuito o de pago) y los formatos de presentación del documento (HTML, texto, PDF o papel), su situación respecto a los derechos de explotación y los ejemplos representativos señalados.

- Cobertura de medios

El número de medios que se incluye en el recurso es un primer elemento de distinción. Se ha podido distinguir entre los sistemas que ofrecen acceso a contenidos informativos de una sola fuente, habitualmente de elaboración propia (hemerotecas digitales de diarios), y los que permiten consultar el contenido de un conjunto más o menos amplio de medios, que serían el resto de tipologías contempladas.

- Alcance temporal

En buena parte de los productos analizados es posible consultar la información retrospectiva y no sólo las noticias del día. Los sistemas más orientados a la información de actualidad son los servicios comerciales de seguimiento de prensa tradicionales y de seguimiento en línea y todos los recursos gratuitos que se describen. Los productos más claramente orientados a la información retrospectiva son los de prensa histórica, las bases de datos de prensa y las hemerotecas de los medios. En algunos casos, como se puede apreciar en la tabla, se ofrecen ambos tipos de información.

- Costo

Los productos orientados al usuario profesional suelen ser de pago, mientras que los dirigidos al público mayoritario son gratuitos, aun 


\begin{tabular}{|c|c|c|c|c|c|c|c|c|c|c|}
\hline 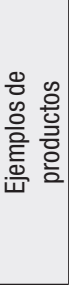 & 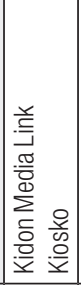 & 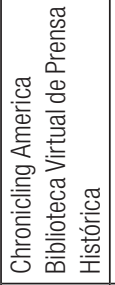 & 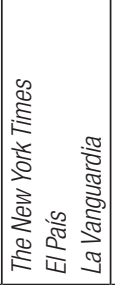 & 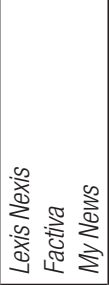 & 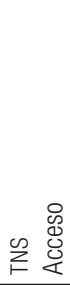 & 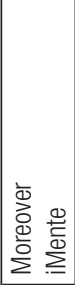 & 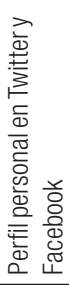 & 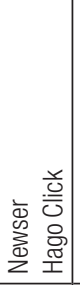 & 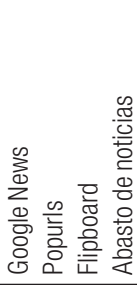 & 음 \\
\hline 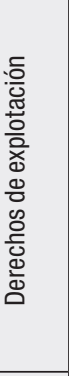 & 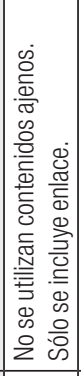 & 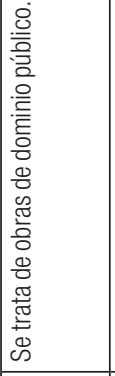 & 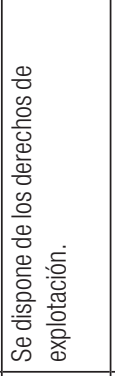 & 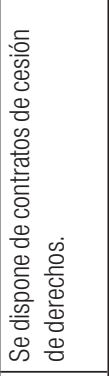 & 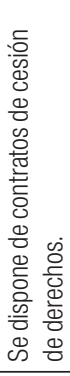 & 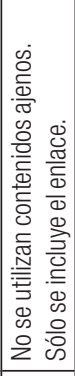 & 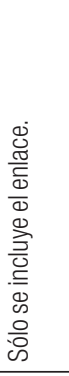 & 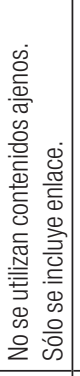 & 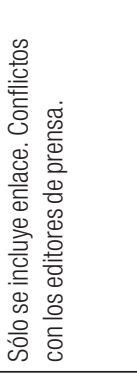 & 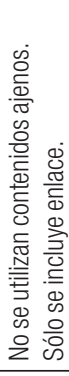 \\
\hline 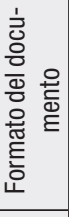 & 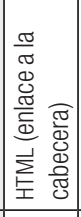 & \begin{tabular}{|l} 
㟔 \\
\end{tabular} & \begin{tabular}{|l} 
\\
㟔 \\
产 \\
至 \\
\end{tabular} & 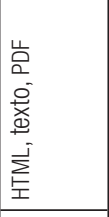 & 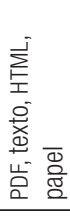 & 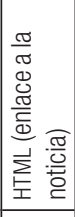 & 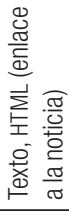 & 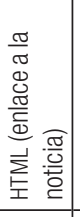 & 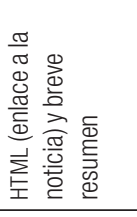 & 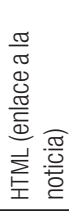 \\
\hline 竎 & 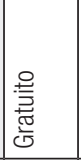 & 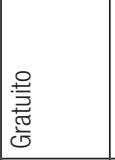 & 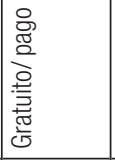 & 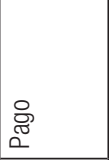 & 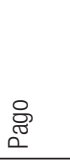 & 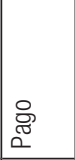 & $\begin{array}{l}\text { 总 } \\
\text { 荧 } \\
\text { 心 }\end{array}$ & 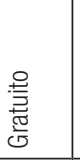 & 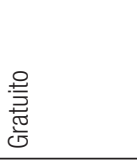 & 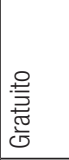 \\
\hline 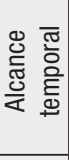 & 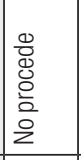 & 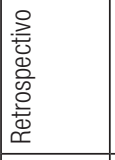 & 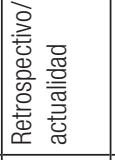 & 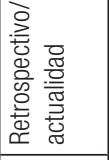 & 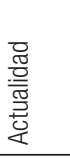 & 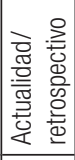 & 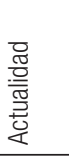 & 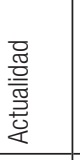 & 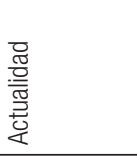 & 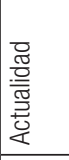 \\
\hline 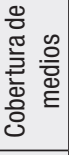 & \begin{tabular}{|l} 
\% \\
喜 \\
\end{tabular} & $\begin{array}{l}\text { \% } \\
\text { 을 } \\
\end{array}$ & $\stackrel{\circ}{5}$ & 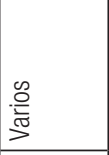 & $\frac{\infty}{\frac{0}{5}}$ & $\begin{array}{l}\text { o } \\
\text { 을 } \\
\end{array}$ & 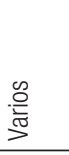 & $\frac{\infty}{\frac{0}{5}}$ & $\frac{\infty}{\frac{0}{5}}$ & 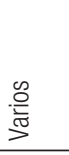 \\
\hline 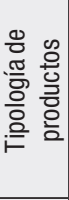 & 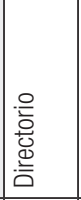 & 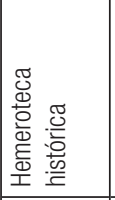 & 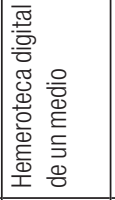 & 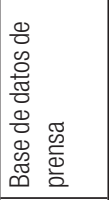 & 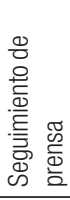 & 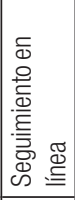 & 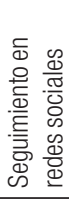 & 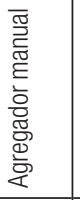 & 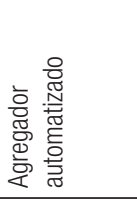 & 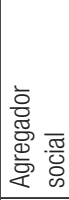 \\
\hline $\begin{array}{l}\text { :은 } \\
\text { 돈 }\end{array}$ & 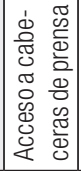 & 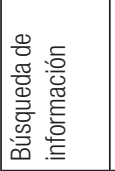 & & & 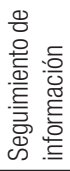 & & & 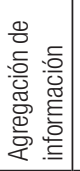 & & \\
\hline
\end{tabular}


cuando éstos también puedan ser de utilidad para profesionales. Las tipologías más claras de sistemas de pago las constituyen las bases de datos de prensa, los servicios de seguimiento tradicionales y los de seguimiento en línea. En una situación mixta (servicios con contenidos de pago y gratuitos) se encuentran las hemerotecas digitales de un solo medio. El resto de los productos son gratuitos.

- Formato de presentación del documento

Las posibilidades de presentación son HTML, texto ASCII, PDF de la edición impresa, papel y enlace a la información original. Este último es el sistema utilizado en la mayoría de productos gratuitos y en los sistemas de seguimiento en línea.

- Derechos de explotación

Algunos de los productos analizados incluyen directamente las informaciones de prensa, ciertos contenidos que son propiedad de las empresas periodísticas que los han elaborado. La relación de los productos analizados respecto de los derechos de explotación se puede resumir en tres opciones. En primer lugar, encontramos aquellos productos que disponen de contratos de cesión de derechos con las empresas periodísticas (es el caso de las bases de datos de prensa o del seguimiento de prensa, que recientemente han aceptado establecer contratos de cesión de derechos). En el otro extremo están aquellos productos sobre los que no procede establecer contrato dado que usan contenidos de dominio público (hemerotecas históricas) o sólo enlazan a la cabecera (directorios). En medio de ambos polos está el caso de los agregadores automáticos (como Google News) que en teoría tan sólo enlazan noticias pero que de acuerdo con algunos editores incluyen también sus contenidos. En este caso, el conflicto sigue abierto y los editores de prensa están pugnando en los juzgados para conseguir una compensación económica.

En lo que se refiere a la evolución y a las perspectivas de crecimiento para cada uno de los productos y sistemas analizados, se observa la presencia de sistemas veteranos ya existentes antes de Internet, que emigraron y se adaptaron en su día a la Web (los clásicos sistemas de directorios, bases de datos de prensa y productos de clipping), junto a los productos aparecidos en los primeros años de la Web (hemerotecas digitales de diarios e históricas fruto de la digitalización, agregadores manuales), los surgidos en la década de 2000 (agregadores automatizados, sistemas de seguimiento en línea, agregadores sociales) y los de aparición muy reciente (servicios de seguimiento en redes sociales y un tipo concreto de agregadores automatizados, los agregadores para dispositivos móviles). 
Mientras algunos sistemas están en declive (agregadores manuales), otros se mantienen en nichos profesionales bien delimitados (bases de datos de prensa, servicios de seguimiento) y otros se encuentran claramente en expansión (agregadores automatizados, seguimiento en redes sociales).

En este sentido, tal vez lo más destacado es que lo que podríamos llamar el ecosistema de la información periodística ha adquirido una gran complejidad en los últimos años, y en buena parte de la mano no solamente de las redes sociales, sino también del actual y creciente protagonismo de la Web móvil. Aunque, como hemos intentado argumentar, cada sistema presenta un esquema distinto y propio de servicios, es indudable que la suma de los dos factores de mayor poder de disrupción -la Web social y la Web móvilabra un nuevo panorama que todavía deberá asentarse en los próximos años.

Se trata en conjunto de un panorama informativo muy dinámico, complejo y cambiante, a cuya caracterización y clarificación hemos querido contribuir. Esperamos por ello que nuestro trabajo haya aportado una propuesta de categorización que permita realizar futuros análisis sobre esta cuestión.

\section{Agradecimientos}

Este trabajo ha contado con el apoyo de la AGAUR, Grupo de Investigación Consolidado "Cultura y Contenidos Digitales" 2009-SGR-177, y de los proyectos del Ministerio español de Economía y Competitividad CSO2012-39518-C04-01 y CSO2012-39518-C04-02.

\section{BibLIOGRAFÍA}

Abadal, Ernest y Guallar, Javier (2010), Prensa digital y bibliotecas, Gijón: Trea.

Arquero Avilés, Rosario y García-Ochoa, María Luisa (2005), La bemeroteca de prensa, Gijón: Trea.

Deuze, Mark (2003), "The web and its journalisms: considering the consequences of different types of newsmedia online", en New Media ES Society, vol. 5, núm. 2, pp. 203-230.

Díaz Noci, Javier (2004), Los resúmenes de prensa en los gabinetes de comunicación: una aproximación jurídica, A Coruña: Netbiblo, 203 pp.

Fuentes, María Eulàlia; Jiménez, Àngels y González, Alfons (2001), "Servicios y sistemas de acceso a la información periodística en Internet", en Anuari de Biblioteconomia, Documentació i Informació: Bibliodoc 2000, Barcelona: COBDC, pp. 85-102. 
Giménez Toledo, Elea (2002), "Las bases de datos para los profesionales de la comunicación”, en Gabriel Galdón (coord.), Teoría y práctica de la documentación informativa, Barcelona: Ariel, pp. 175-196.

Giménez Toledo, Elea y López Martín, Laura (2003), "Fuentes de información especializada: sobre periodismo y para periodistas", en Víctor Manuel Pareja Pérez (coord.), Guía de Internet para periodistas, Madrid: Centro de Información y Documentación Cindoc, pp. 107-150.

González, Alfons (2009), "Evolución y estado actual de las bases de datos de noticias en internet”, en Xose López García y Rosa Aneiros Díaz (eds.), Documentacion e poder na sociedade da información: o papel dos arquivos e os observatorios da comunicación, Consello da Cultura Galega, pp. 165-186.

Guallar, Javier y Abadal, Ernest (2009a), "Evaluación de hemerotecas de prensa digital: indicadores y ejemplos de buenas prácticas", en El profesional de la información, vol. 18, núm. 3, pp. 255-269, disponible en: http://eprints.rclis.org/13048/ (Fecha de consulta: 16 de enero de 2013).

Guallar, Javier y Abadal, Ernest (2009b), "Fuentes de información sobre prensa digital: una propuesta de clasificación”, en I Congreso internacional de ciberperiodismo y web 2.0, Bilbao, 11-13 noviembre, disponible en http://eprints.rclis.org/13767/ (Fecha de consulta: 16 de enero de 2013).

Guallar, Javier y Abadal, Ernest (2010), “The digital press archives of the leading Spanish online newspapers", en Information research, vol. 15, núm. 1, disponible en: http://hdl.handle.net/10760/14439 (Fecha de consulta: 16 de enero de 2013).

Guallar, Javier; Abadal, Ernest y Codina, Lluís (2012), "Hemerotecas de prensa digital: evolución y tendencias", en El profesional de la información, vol. 21, núm. 6, pp. 595-605, disponible en: http:// eprints.rclis.org/18199/ (Fecha de consulta: 16 de enero de 2013).

Guallar, Javier y Masip, Pere (2012), "Lectura de prensa en dispositivos móviles”, en José Antonio Cordón García et al. (coord.), Libros electrónicos y contenidos digitales en la sociedad del conocimiento: mercado, servicios y derechos, Madrid: Pirámide, pp. 219236.

Guallar, Javier y Redondo, Sílvia (2010), "Fonts d'informació professionals de premsa. Una panoràmica”, en 12es Jornades Catalanes d'Informació i Documentació, Barcelona, 19-20 mayo, disponible en: http://eprints.rclis.org/handle/10760/14644 (Fecha de consulta: 16 de enero de 2013).

Ivars-Nicolás, Begoña (2012), "Canales alternativos de acceso a la información en la prensa española en internet. La noticia fuera del periódico”, en El profesional de la información, vol. 21, núm. 4, pp. 396-400. 
Jiménez López, Àngels (2002), Estudi de la gestió documental de la informació en els serveis de valor afegit dels mitjans de comunicació a internet: el cas de la premsa diària a l'estat español, Tesi doctoral, Universitat Autònoma de Barcelona, disponible en: http://www. tdx.cat/handle/10803/4169 (Fecha de consulta: 16 de enero de 2013).

Jiménez, Àngels (2003), "Acceso a información periodística a través de servicios de press clipping”, en Hipertext, núm. 1, disponible en: http://www.upf.edu/hipertextnet/numero-1/press-clipping.html (Fecha de consulta: 16 de enero de 2013).

Palacios, Marcos (2009), "La memoria como criterio de valoración de calidad en el ciberperiodismo: algunas consideraciones", en El profesional de la información, vol. 18, núm. 3, pp. 270-276.

Pareja Pérez, Víctor Manuel (coord.) (2003), Guía de Internet para periodistas, Madrid: Centro de Información y Documentación Cindoc (CSIC), 196 pp.

Pareja Pérez, Víctor Manuel (2006), "Periodismo digital. Medios de comunicación en Internet”, en Ángeles Maldonado y Luis Rodríguez Yunta (coords.), La información especializada en internet: directorio de recursos de interés académico y profesional, Madrid: CSIC, pp. 237-276.

Renedo, José Ángel (2006), "Buscadores de noticias versus prensa en Internet”, en Buscanoticias, disponible en: http://buscanoticias. blogspot.com/2006/01/buscadores-de-noticias-versus-prensa.html (Fecha de consulta: 16 de enero de 2013).

Rubio Lacoba, María (2002), "Otras fuentes en Internet”, en Gabriel Galdón (coord.), Teoría y práctica de la documentación informativa, Barcelona: Ariel, pp. 175-196.

Sánchez Vigil, Juan Miguel; Marcos Recio, Juan Carlos y Olivera Zaldua, María (2011), "La nueva ecología de los documentos en la sociedad del conocimiento: aportaciones al proyecto Infoscopos", en Actas del VIII Seminario Hispano-Mexicano de Biblioteconomía y Documentación, pp. 163-172.

Varela, Juan (2012), “¿Pueden abandonar Google los diarios? Un negocio para la fragmentación del mercado", en Periodistas 21, 26 octubre 2012, disponible en: http://periodistas21.blogspot.com. es/2012/10/pueden-abandonar-google-los-diarios-un.html (Fecha de consulta: 16 de enero de 2013).

Zamith, Fernando (2008), Ciberjornalismo. As potencialidades da Internet nos sites noticiosos portugueses, Porto: Ediçoes Afrontamento. 